\title{
Ten-year follow-up results of perioperative chemotherapy with doxorubicin and ifosfamide for high-grade soft-tissue sarcoma of the extremities: Japan Clinical Oncology Group study JCOG0304
}

\author{
Kazuhiro Tanaka ${ }^{1 *}$ (D, Junki Mizusawa ${ }^{2}$, Norifumi Naka ${ }^{3}$, Akira Kawai ${ }^{4}$, Hirohisa Katagiri ${ }^{5}$, Toru Hiruma ${ }^{6}$, \\ Yoshihiro Matsumoto ${ }^{7}$, Hiroyuki Tsuchiya ${ }^{8}$, Robert Nakayama ${ }^{9}$, Hiroshi Hatano ${ }^{10}$, Makoto Emori ${ }^{11}$, \\ Munenori Watanuki ${ }^{12}$, Yukihiro Yoshida ${ }^{13}$, Takeshi Okamoto ${ }^{14}$, Satoshi Abe ${ }^{15}$, Kunihiro Asanuma ${ }^{16}$, \\ Ryohei Yokoyama ${ }^{17}$, Hiroaki Hiraga ${ }^{18}$, Tsukasa Yonemoto ${ }^{19}$, Takeshi Morii ${ }^{20}$, Keisuke Ae ${ }^{21}$, Akihito Nagano ${ }^{22}$, \\ Hideki Yoshikawa ${ }^{23}$, Haruhiko Fukuda ${ }^{2}$, Toshifumi Ozaki ${ }^{24}$ and Yukihide Iwamoto ${ }^{25}$
}

\begin{abstract}
Background: Soft-tissue sarcomas (STS) are rare malignant tumors those are resistant to chemotherapy. We have previously reported the 3-year follow-up result on the efficacy of perioperative chemotherapy with doxorubicin (DXR) and ifosfamide (IFM) for high-risk STS of the extremities (JCOG0304). In the present study, we analyzed the 10-year follow-up results of JCOG0304.
\end{abstract}

Methods: Patients with operable, high-risk STS (T2bNOMO, AJCC 6th edition) of the extremities were treated with 3 courses of preoperative and 2 courses of postoperative chemotherapy, which consisted of $60 \mathrm{mg} / \mathrm{m}^{2}$ of DXR plus $10 \mathrm{~g} / \mathrm{m}^{2}$ of IFM over a 3-week interval. The primary study endpoint was progression-free survival (PFS) estimated by Kaplan-Meier methods. Prognostic factors were evaluated by univariable and multivariable Cox proportional hazards model.

Results: A total of 72 patients were enrolled between March 2004 and September 2008, with 70 of these patients being eligible. The median follow-up period was 10.0 years for all eligible patients. Local recurrence and distant metastasis were observed in 5 and 19 patients, respectively. The 10-year PFS was 65.7\% (95\% Cl: 53.4-75.5\%) with no PFS events being detected during the last 5 years of follow-up. The 10 -year overall survival was $78.1 \%$ (95\% Cl: 66.3-86.2\%). Secondary malignancy was detected in 6 patients. The subgroup analysis demonstrated that there was significant difference in survival with regard to primary tumor size.

Conclusions: Only a few long-term results of clinical trials for perioperative chemotherapy treatment of STS have been reported. Our results demonstrate that the 10-year outcome of JCOG0304 for patients with operable, high-risk STS of the extremities was stable and remained favorable during the last 5 years of follow-up.

Trial registration: This trial was registered at the UMIN Clinical Trials Registry as C000000096 on August 30, 2005.

Keywords: Soft tissue sarcoma, Extremity, Perioperative chemotherapy, Doxorubicin and ifosfamide, 10-year followup, Survival

\footnotetext{
* Correspondence: ktanaka@oita-u.ac.jp

${ }^{1}$ Department of Orthopaedic Surgery, Faculty of Medicine, Oita University,

Idaigaoka 1-1, Hasama, Yufu City, Oita 879-5593, Japan

Full list of author information is available at the end of the article
}

(c) The Author(s). 2019 Open Access This article is distributed under the terms of the Creative Commons Attribution 4.0 International License (http://creativecommons.org/licenses/by/4.0/), which permits unrestricted use, distribution, and reproduction in any medium, provided you give appropriate credit to the original author(s) and the source, provide a link to the Creative Commons license, and indicate if changes were made. The Creative Commons Public Domain Dedication waiver (http://creativecommons.org/publicdomain/zero/1.0/) applies to the data made available in this article, unless otherwise stated. 


\section{Background}

Soft-tissue sarcomas (STS) are rare types of malignant tumors those account for less than $1 \%$ of all malignancies [1]. According to the Soft Tissue Tumor Registry conducted by the Japanese Orthopaedic Association, 1529 patients with STS were registered in 2015 in Japan [2]. The standard treatment for localized, low-grade and/or small STS is surgery. On the other hand, highgrade, large, and deep-seated STS are considered to be high-risk and adjuvant chemotherapy could be a treatment option for this type of STS [3].

We conducted a multicenter phase 2 trial (JCOG0304) of perioperative chemotherapy with doxorubicin (DXR) and ifosfamide (IFM) for treating high-risk STS of the extremities [4]. We have previously reported the perioperative chemotherapy with DXR and IFM was well tolerable and promising in the short-term results of JCOG0304, including the efficacy, safety, and correlation of outcomes with prognostic factors and radiological response [5-7]. In the present study, we analyzed the 10year follow-up results and report the final results of JCOG0304.

\section{Methods}

From March 2004 to September 2008, a total of 72 patients with high-risk (i.e. high-grade, deep-seated tumors with size $>5 \mathrm{~cm}$ ), operable STS of the extremities were enrolled in the phase II trial JCOG0304. The study protocol of JCOG0304 was approved by the Protocol Review Committee of the Japan Clinical Oncology Group (JCOG) and was also approved by the Institutional Review Boards of each of the 27 participating institutions. Written informed consent was obtained from each patient before study enrollment.

Details for the eligibility criteria of JCOG0304 have been reported elsewhere [5]. Briefly, the main inclusion criteria were: (1) histologically proven STS of stage III (T2bN0M0), according to the AJCC/UICC 6th edition [8]; (2) histological grade of 2 or 3, according to the French Federation of Cancer Center (FNCLCC) system [9]; (3) resectable tumor in the extremities; (4) age $\geq 20$ years, but $\leq 70$ years; (5) Eastern Cooperative Oncology Group (ECOG) performance status 0 or 1 ; and (6) sufficient organ function. The histological diagnosis of biopsy specimens from all patients was reviewed after patient registration by an independent central pathologic committee, which consisted of 3 pathologists who specialized in diagnosing STS.

Preoperative chemotherapy was administrated for 3 courses, followed by surgical resection, and 2 additional courses of postoperative chemotherapy.

Each course of chemotherapy was given every 3 weeks and consisted of DXR $\left(30 \mathrm{mg} / \mathrm{m}^{2} /\right.$ day on days 1 and 2) and IFM ( $2 \mathrm{~g} / \mathrm{m}^{2} /$ day on days $\left.1-5\right)$. Mesna $(1200 \mathrm{mg} /$ $\mathrm{m}^{2}$ /day) was added after the administration of IFM on days 1-5. Granulocyte-colony stimulating factor was used prophylactically for 7 days if the patient experienced grade 4 neutropenia or leukopenia for more than 5 days after chemotherapy in the previous course.

Surgical treatment was carried out within 5 weeks of the last administration of preoperative chemotherapy. A wide margin should be attempted for the tumor resection; however, a marginal margin was also allowed. If the surgical margin was assessed as insufficient (i.e., margin $<1 \mathrm{~cm}$ ), a marginal or intralesional margin and local radiation therapy was permitted after the protocol treatment. The decision of administration of radiation was depend on discretionary of the treating physicians, thus the details of radiation therapy were not defined in the protocol of JCOG0304.

The JCOG Data Center conducted data management and the statistical analysis of the present study. Details of the criteria for radiological and pathological response evaluation in JCOG0304 have been reported [5]. Adverse events were evaluated using the National Cancer Institute Common Toxicity Criteria (version 2.0).

\section{Endpoints and statistical analysis}

The primary endpoint of the JCOG0304 study was 2year progression-free survival (PFS), with secondary endpoints of radiological and pathological response to preoperative chemotherapy, overall survival (OS), and adverse events. PFS and OS were defined as the time from enrollment to disease progression or death, and to death, respectively. The PFS and OS were estimated by the Kaplan-Meier method. Univariable and multivariable Cox regression analyses were conducted to investigate the prognostic impact of variables on PFS, which included age, sex, histological subtype, tumor differentiation, necrosis, mitosis, histological grade by FNCLCC and Ki-67 system [6], and tumor size. Data assessed by institutional decision was used for univariable analysis only, whereas the data reviewed by the Central Pathological Committee was used for univariable and multivariable analyses. Hazard ratios and $p$ values were calculated by Cox regression model. As a sensitivity analysis, multivariable analysis with backward elimination method with alpha of 0.2 was also performed. The difference was considered as significant if the $p$ value was $<0.05$. All statistical analyses were carried out using SAS software version 9.1 or higher (SAS Institute, Cary, NC, USA).

\section{Results}

\section{Patients and treatment}

Details of the characteristics of the 72 enrolled and 70 eligible patients have been reported elsewhere [5]. The median follow-up period of all eligible patients 
beginning from enrollment was 10.0 years (interquartile range, 2.1-11.6 years). The median follow-up duration of the 54 patients without progression or death was 10.4 years (interquartile range, 9.3-to 12.0 years). A diagram of the study flow is shown in Fig. 1. Three courses of preoperative chemotherapy were completed in 66 patients and all 5 courses of the protocol treatment were completed in 53 patients. For local treatment, all eligible patients underwent surgery and 12 patients were treated by adjuvant radiation therapy as a post-protocol treatment.

\section{Efficacy and safety}

When the trial database closed in October 2018, 16 patients had died, with 19 and 5 patients having developed distant and local relapse, respectively. In 12 patients treated by adjuvant radiation therapy, no local recurrence was observed. All 5 patients who had local recurrence were treated by negative margin in surgical resection, and one out of the five patients had died. Compared to the previous reports on JCOG0304 with a median follow-up of 5.1 years, 4 more patients died. In addition, no local or distant relapse were observed

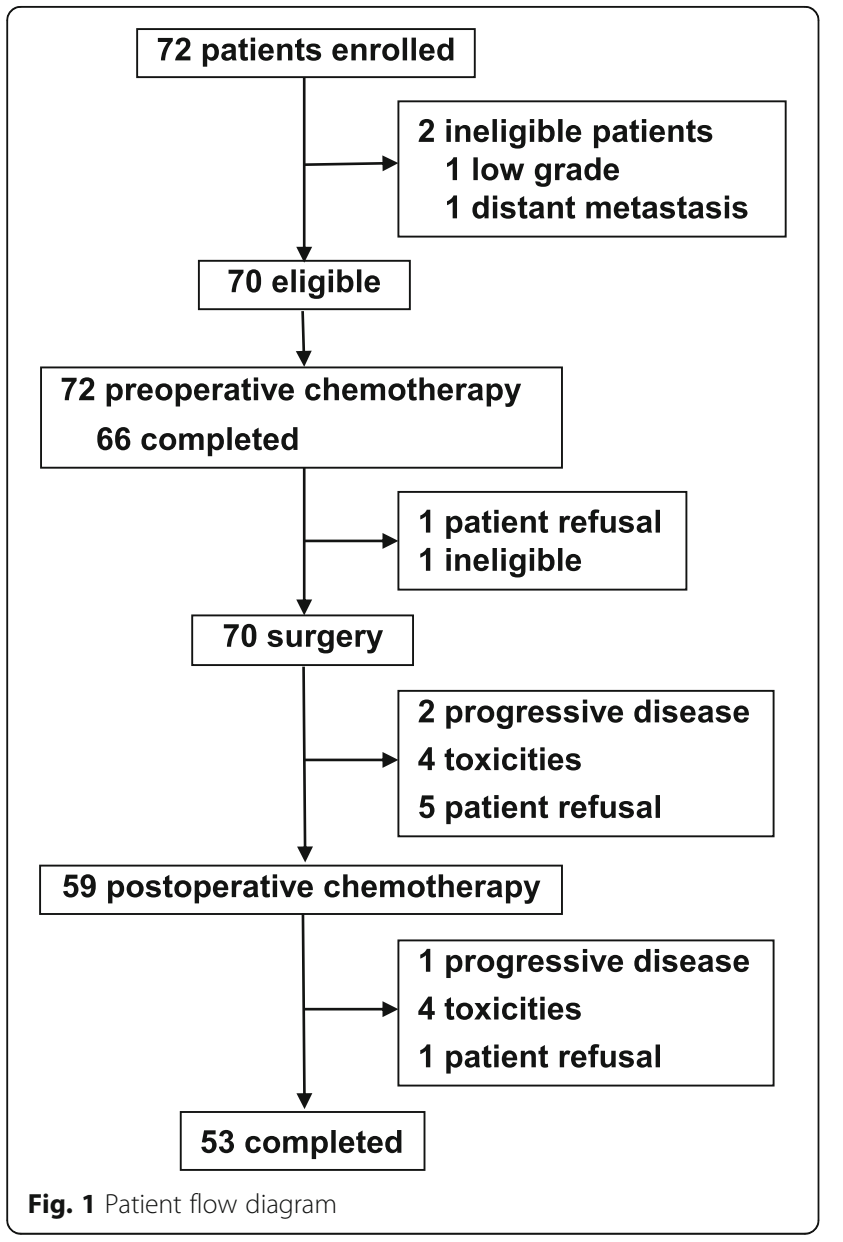

within the following 5 years after the data cut-off date for the previous reports. For the 70 eligible cases, the 5and 10-year OS was $82.9 \%$ (95\% CI: $71.8-89.9 \%)$ and 78.1\% (95\% CI, 66.3-86.2\%), respectively (Fig. 2). Furthermore, the 5- and 10-year PFS were both $65.7 \%$ (95\% CI: 53.4-75.5\%) (Fig. 3).

No treatment related death was observed in the present study. Secondary malignancies were found in 6 patients (breast cancer, chronic myeloid leukemia, prostate cancer, sigmoid colon cancer, lung cancer, gastric malignant lymphoma), however, all of these were considered to be unrelated to the protocol treatment. Regarding late adverse effects, grade 3 ventricular arrhythmia was observed in only 1 patient. Grades 1 and 2 left ventricular dysfunction was observed in 1 patient each. Grade 1 edema, as well as grades 1 and 2 peripheral nerve dysfunction, were found in 5,4 , and 1 patients, respectively.

In the univariable analysis, there were no significant differences in PFS regarding sex (male vs. female), age ( $<40$ vs. $\geq 40$ years), ECOG performance status ( 0 vs. 1 ), histological subtype (undifferentiated pleomorphic sarcoma, leiomyosarcoma, synovial sarcoma, or other), histological tumor differentiation (score 3 vs. 2), tumor necrosis (score 1 or 2 vs. 0), and mitosis (score 2 or 3 vs. 1), histological grade evaluated by FNCLCC system (grade 3 vs. 2) and Ki-67 system (grade 3 vs. 2). However, primary tumor size $(\leq 10 \mathrm{~cm}$ vs. $>10 \mathrm{~cm})$ significantly influenced on PFS of the patients (Table 1). Furthermore, the multivariable analyses both with all variables and backward elimination demonstrated that there was significant difference in PFS with regard to the tumor size (Fig. 4, Table 2).

\section{Discussion}

In the present study, we focused on the long-term results of JCOG0304, as well as the correlations between prognostic factors and outcomes, at a median follow-up of 10 years. Only a few long-term results of clinical trials studying perioperative chemotherapy for STS have been reported due to time and cost issues $[10,11]$. As in our previous report, the favorable outcomes of JCOG0304 were maintained during the long-term follow-up of 10 years. We found that perioperative chemotherapy with DXR plus IFM might be useful as a treatment for highrisk STS of the extremities.

In previous clinical trials of adjuvant chemotherapy for STS, it was known that local and distant failure may appear, even as long as 5 years after treatment, and that long-term results of adjuvant chemotherapy tend to lose their initial advantage in survival over surgery alone. For instance, in a randomized controlled trial (RCT) conducted by the Italian Sarcoma Group (ISG) on epirubicin (EPI) and IFM, which compared adjuvant 


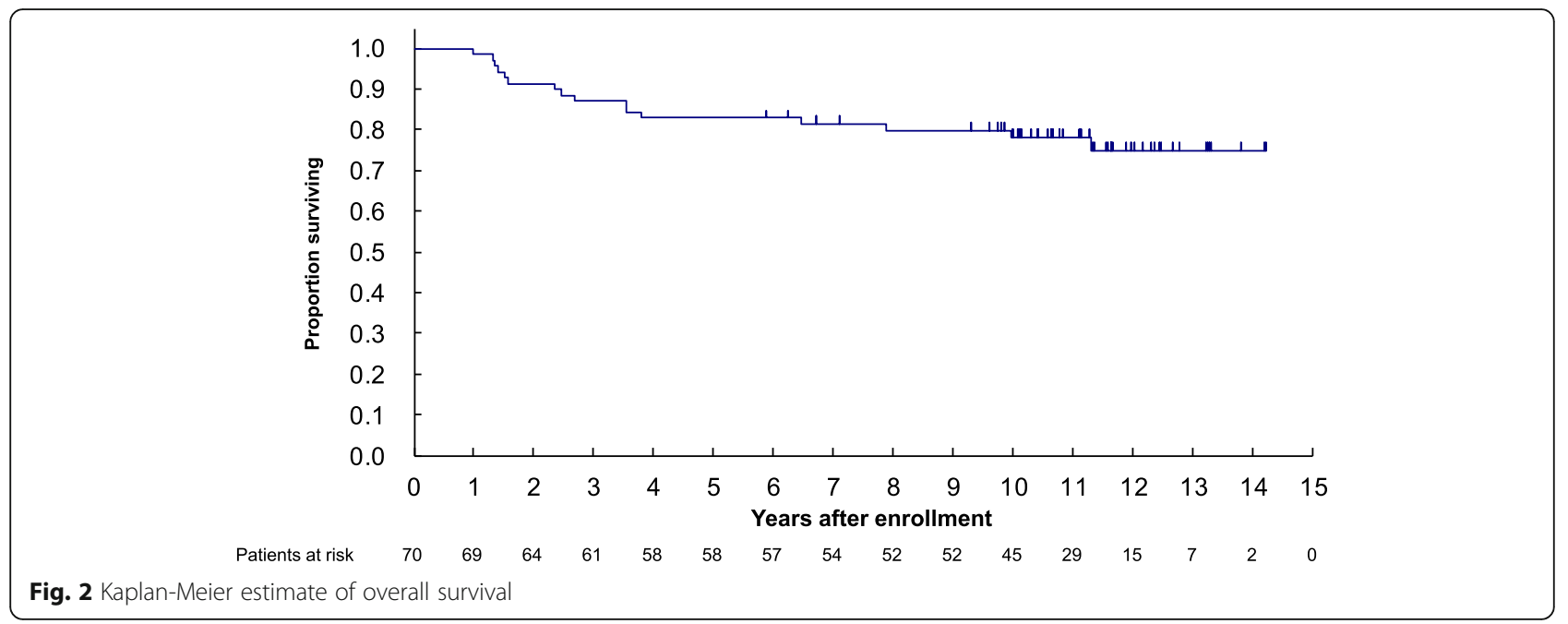

chemotherapy and surgery alone, the 4-year OS of the adjuvant chemotherapy arm was significantly better than that of the surgery alone arm [12]. However, this superiority was lost in subsequent long-term observation and the effectiveness of postoperative adjuvant chemotherapy was considered to be questionable [13].

The ISG conducted another RCT on full-dose EPI and IFM by comparing 5 total courses of pre- and post-operative chemotherapy with 3 courses of preoperative chemotherapy. This trial in which 328 patients with high-risk STS of the extremities and trunk were registered, demonstrated the non-inferiority of the 3-course preoperative chemotherapy for survival [14]. From the long-term observation of the trial, the good 5-year survival results were maintained at the 10-year follow-up [10]. Both the trial of neoadjuvant chemotherapy by ISG and the JCOG0304 study showed long-term stable results. The commonality of both studies is that 3 courses of full-dose anthracycline and IFM have been used prior to surgery. In addition, $91.7 \%$ of enrolled patients could complete 3 courses of preoperative chemotherapy. Although both studies lacked a surgery alone arm, the results suggest that preoperative chemotherapy with anthracycline and IFM might be highly effective for treating high-risk STS.

Regarding the usefulness of preoperative chemotherapy for treating high-risk STS, the ISG-STS1001 RCT, which included a 3-course chemotherapy regimen in the neoadjuvant setting, showed interesting results [15]. In this trial, full-dose EPI and IFM as the standard treatment arm and histology-tailored regimens as the experimental arm were compared. The results demonstrated a significantly better 4-year disease-free survival in the standard arm than that of the experimental arm (62 and $38 \%$, respectively, $p=0.006$ ), as well as 4 -year OS (89 and $64 \%$, respectively, $p=0.034$ ). The results in the standard arm were similar to those of the JCOG0304 trial, while those of the histology-tailored chemotherapy

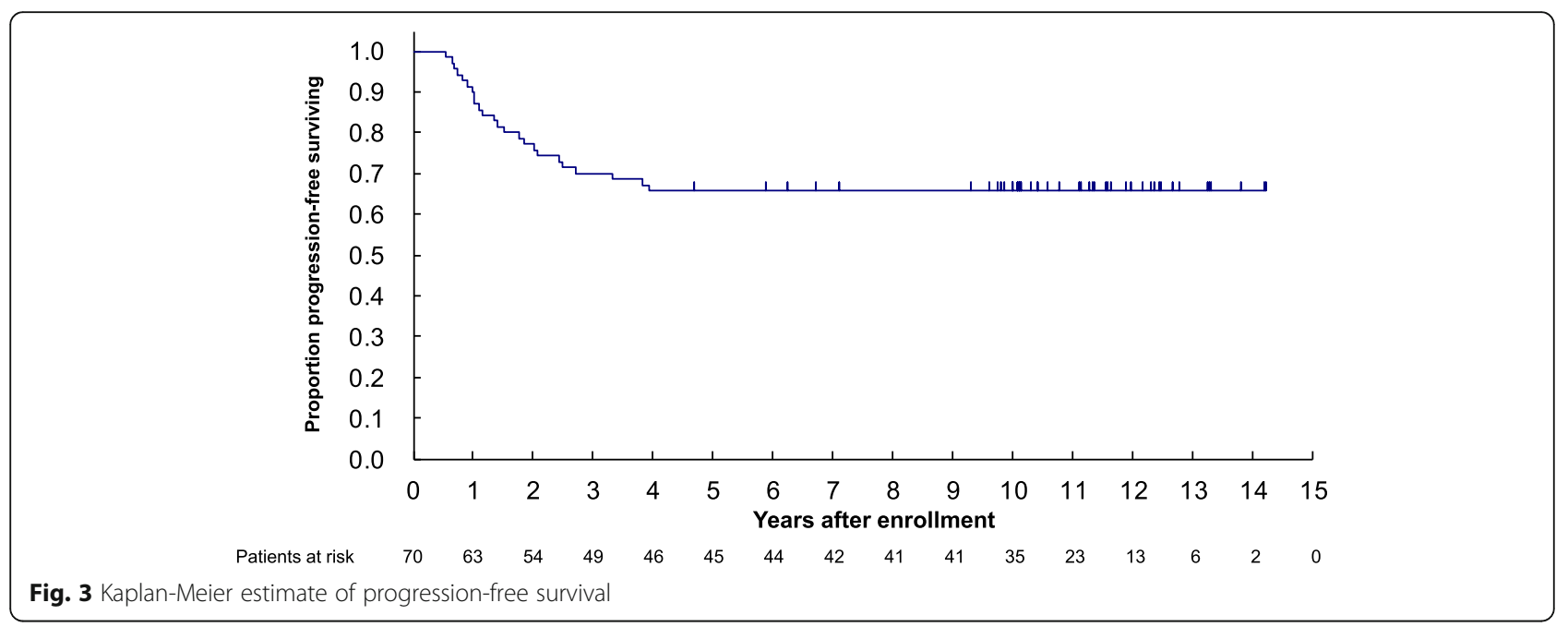


Table 1 Univariable analysis for progression-free survival

\begin{tabular}{|c|c|c|c|}
\hline \multicolumn{4}{|l|}{ Univariable } \\
\hline \multirow[t]{2}{*}{ Factors } & \multirow[t]{2}{*}{ Category } & \multicolumn{2}{|c|}{ Univariable analysis } \\
\hline & & HR $(95 \% \mathrm{Cl})$ & $\begin{array}{l}p- \\
\text { value }\end{array}$ \\
\hline Sex & Female (vs. Male) & $\begin{array}{l}0.61(0.27- \\
1.38)\end{array}$ & 0.23 \\
\hline Age (Years) & $\geq 40$ (vs. < 40) & $\begin{array}{l}2.04(0.76- \\
5.47)\end{array}$ & 0.16 \\
\hline Performance Status & 1 (vs. 0) & $\begin{array}{l}1.11(0.46- \\
2.69)\end{array}$ & 0.81 \\
\hline \multirow[t]{3}{*}{ Histological subtype } & $\begin{array}{l}\text { Leiomyosarcoma (vs. } \\
\text { UPS) }\end{array}$ & $\begin{array}{l}0.59(0.18- \\
1.98)\end{array}$ & 0.40 \\
\hline & $\begin{array}{l}\text { Synovial sarcoma (vs. } \\
\text { UPS) }\end{array}$ & $\begin{array}{l}0.34(0.10- \\
1.12)\end{array}$ & 0.08 \\
\hline & Others (vs. UPS) & $\begin{array}{l}0.64(0.24- \\
1.72)\end{array}$ & 0.38 \\
\hline Tumor differentiation & 3 (vs. 2) & $\begin{array}{l}0.64(0.29- \\
1.43)\end{array}$ & 0.28 \\
\hline Tumor necrosis & 1 or 2 (vs. 0) & $\begin{array}{l}1.28(0.56- \\
2.93)\end{array}$ & 0.56 \\
\hline Tumor mitosis & 2 or 3 (vs. 1) & $\begin{array}{l}1.14(0.51- \\
2.57)\end{array}$ & 0.75 \\
\hline $\begin{array}{l}\text { Histological grade } \\
\text { (FNCLCC) }\end{array}$ & Grade 3 (vs. 2) & $\begin{array}{l}0.94(0.42- \\
2.09)\end{array}$ & 0.87 \\
\hline $\begin{array}{l}\text { Histological grade (Ki- } \\
67)\end{array}$ & Grade 3 (vs. 2) & $\begin{array}{l}1.38(0.61- \\
3.12)\end{array}$ & 0.43 \\
\hline Tumor size & $>10 \mathrm{~cm}(\mathrm{vs} . \leq 10 \mathrm{~cm})$ & $\begin{array}{l}3.15(1.37- \\
7.21)\end{array}$ & 0.0067 \\
\hline
\end{tabular}

Abbreviations: $\mathrm{HR}$ hazard ratio, $\mathrm{Cl}$ confidence interval, UPS, undifferentiated pleomorphic sarcoma, FNCLCC French Federation of Cancer Center

arm were similar to those of the surgery alone arm in the study of Frustaci et al. [12]. Moreover, univariable and multivariable analyses in JCOG0304 demonstrated no significant differences in outcomes regarding histological subtype (undifferentiated pleomorphic sarcoma, leiomyosarcoma, synovial sarcoma, or other). So far, the standard treatment with anthracycline and IFM might be favorable for STS of major histological subtypes, at least for those eligible in JCOG0304 and ISG-STS1001.

Since ISG-STS1001 study had a short median followup time of 12.3 months, long-term observation is required to confirm these findings. However, compared to ineffective chemotherapy (or surgery alone), the results of the ISG-STS1001 suggested that an improved survival proportion of approximately $20 \%$ can be expected for the patients with high-risk STS who receive full-dose anthracycline plus IFM combination chemotherapy administered before surgery [15]. Regarding the efficacy of perioperative chemotherapy for treating STS, a metaanalysis of 18 RCTs that compared surgery alone and adjuvant chemotherapy was performed. The absolute risk reduction for death by adjuvant chemotherapy was found to be $6 \%$ (95\% CI: $2-11 \%$ ), while that of DXR plus IFM combination chemotherapy was $11 \%$ (95\% CI: $3-$ $19 \%$ ), which demonstrates the usefulness of adding IFM to DXR regimens [16].

After the above-mentioned meta-analysis, the European Organization for Research and Treatment on Cancer (EORTC) conducted a large-scale RCT, EORTC62931, which compared surgery alone and an adjuvant chemotherapy regimen of DXR plus IFM for the treatment of STS. No significant difference between the 2 groups in terms of OS was observed in the trial, which raised doubts regarding the efficacy of adjuvant chemotherapy for treating STS [17]. However, there were several concerns regarding the interpretation of the results of the above trial. In EORTC62931, the IFM dose was low $\left(5 \mathrm{~g} / \mathrm{m}^{2}\right)$, which was only half that of the ISG trials and JCOG0304. The study enrolled patients with STS of any site or size contained patients (24\%) with small tumors $(<5 \mathrm{~cm}$, minimum $0.4 \mathrm{~cm})$. Given these

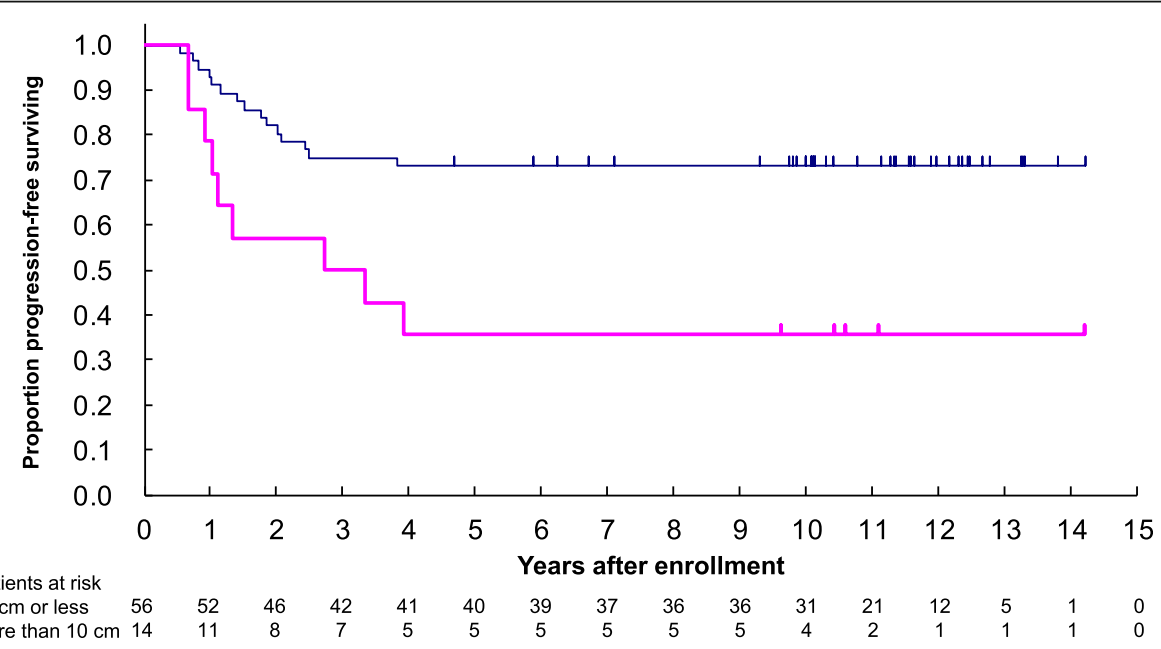

Fig. 4 Kaplan-Meier estimate of progression-free survival by primary tumor size 
Table 2 Multivariable analysis for progression-free survival

\begin{tabular}{|c|c|c|c|}
\hline \multicolumn{4}{|l|}{ Multivariable } \\
\hline \multirow[t]{2}{*}{ Factors } & \multirow[t]{2}{*}{ Category } & \multicolumn{2}{|c|}{$\begin{array}{l}\text { Multivariable analysis } \\
\text { (including all } \\
\text { variables) }\end{array}$} \\
\hline & & HR $(95 \% \mathrm{Cl})$ & $\begin{array}{l}p^{-} \\
\text {value }\end{array}$ \\
\hline Sex & Female (vs. Male) & $\begin{array}{l}0.70(0.29- \\
1.72)\end{array}$ & 0.44 \\
\hline Age (Years) & $\geq 40$ (vs. $<40)$ & $\begin{array}{l}1.47(0.47- \\
4.58)\end{array}$ & 0.50 \\
\hline Performance Status & 1 (vs. 0) & $\begin{array}{l}1.41(0.56- \\
3.59)\end{array}$ & 0.47 \\
\hline \multirow[t]{3}{*}{ Histological subtype } & $\begin{array}{l}\text { Leiomyosarcoma (vs. } \\
\text { UPS) }\end{array}$ & $\begin{array}{l}0.61(0.17- \\
2.18)\end{array}$ & 0.44 \\
\hline & $\begin{array}{l}\text { Synovial sarcoma (vs. } \\
\text { UPS) }\end{array}$ & $\begin{array}{l}0.48(0.13- \\
1.80)\end{array}$ & 0.28 \\
\hline & Others (vs. UPS) & $\begin{array}{l}0.65(0.22- \\
1.87)\end{array}$ & 0.42 \\
\hline $\begin{array}{l}\text { Histological grade } \\
\text { (FNCLCC) }\end{array}$ & Grade 3 (vs. 2) & $\begin{array}{l}0.56(0.17- \\
1.87)\end{array}$ & 0.34 \\
\hline $\begin{array}{l}\text { Histological grade (Ki- } \\
67 \text { ) }\end{array}$ & Grade 3 (vs. 2) & $\begin{array}{l}1.72(0.51- \\
5.80)\end{array}$ & 0.39 \\
\hline Tumor size & $>10 \mathrm{~cm}(\mathrm{vs} . \leq 10 \mathrm{~cm})$ & $\begin{array}{l}2.85(1.18- \\
6.92)\end{array}$ & 0.0203 \\
\hline \multirow[t]{2}{*}{ Factors } & \multirow[t]{2}{*}{ Category } & \multicolumn{2}{|c|}{$\begin{array}{l}\text { Multivariable analysis } \\
\text { (backward } \\
\text { elimination with } \\
\text { alpha }=20 \% \text { ) }\end{array}$} \\
\hline & & HR $(95 \% \mathrm{Cl})$ & $\begin{array}{l}p- \\
\text { value }\end{array}$ \\
\hline Sex & Female (vs. Male) & & \\
\hline Age (Years) & $\geq 40$ (vs. $<40$ ) & & \\
\hline Performance Status & 1 (vs. 0) & & \\
\hline \multirow[t]{3}{*}{ Histological subtype } & $\begin{array}{l}\text { Leiomyosarcoma (vs. } \\
\text { UPS) }\end{array}$ & & \\
\hline & $\begin{array}{l}\text { Synovial sarcoma (vs. } \\
\text { UPS) }\end{array}$ & & \\
\hline & Others (vs. UPS) & & \\
\hline $\begin{array}{l}\text { Histological grade } \\
\text { (FNCLCC) }\end{array}$ & Grade 3 (vs. 2) & $\begin{array}{l}0.42(0.13- \\
1.34)\end{array}$ & 0.14 \\
\hline $\begin{array}{l}\text { Histological grade (Ki- } \\
67)\end{array}$ & Grade 3 (vs. 2) & $\begin{array}{l}2.39(0.75- \\
7.62)\end{array}$ & 0.14 \\
\hline Tumor size & $>10 \mathrm{~cm}(\mathrm{vs} . \leq 10 \mathrm{~cm})$ & $\begin{array}{l}3.27(1.41- \\
7.59)\end{array}$ & 0.0057 \\
\hline
\end{tabular}

Abbreviations: $H R$ hazard ratio, $\mathrm{Cl}$ confidence interval, UPS undifferentiated pleomorphic sarcoma, FNCLCC French Federation of Cancer Center

observations, the most useful perioperative chemotherapy regimen currently available is full-dose DXR and IFM combination therapy, and population that would most benefit from this therapy might be high-risk STS of the limb and trunk.

The limitation of the present study is that the JCOG0304 trial was a single-arm phase 2 study and no direct comparison with surgery or postoperative chemotherapy alone was examined. In addition, the number of registered patients was only 72 , which can limit the impact of our results.

Taken together with the 10-year results of the present study and the ISG trial, preoperative chemotherapy using full-dose anthracycline and IFM is considered to be useful for treating high-risk STS of the extremities and trunk. It may also be necessary to wait for the final results of the ISG-STS1001 trial. At present, we are conducting a randomized phase $2 / 3$ study of perioperative chemotherapy for the treatment of high-risk STS arising in the extremities and trunk, by comparing DXR plus IFM with gemcitabine plus docetaxel, JCOG1306 [18]. In JCOG1306, we aim to confirm non-inferiority of gemcitabine plus docetaxel to DXR plus IFM since the former regimen is less toxic and has a possibility of combination with other drug including molecular-target therapy in the future therapeutic development.

\section{Conclusions}

The JCOG0304 trial showed a favorable, long-term efficacy of perioperative chemotherapy for treating high-risk STS of the extremities. DXR and IFM might be the most useful perioperative chemotherapy regimen for treating STS. To maximize treatment benefit, full-dose anthracycline plus IFM for patients with high-grade, large and deep-seated STS located in the limbs and trunk may be useful.

\section{Additional file}

Additional file 1: Table S1. Names of the Institutional Review Boards of participating institutions. (DOCX $12 \mathrm{~kb}$ )

\author{
Abbreviations \\ Cl: Confidence interval; DXR: doxorubicin; EORTC: European Organisation for \\ Research and Treatment of Cancer; IFM: ifosfamide; JCOG: Japan Clinical \\ Oncology Group; OS: Overall survival; PFS: Progression-free survival; STS: Soft-
} tissue sarcoma

\begin{abstract}
Acknowledgements
The abstract of the present study has been presented at the 2019 American Society of Clinical Oncology Annual Meeting (Abstract No: e22533) [19]. We would like to thank Editage (www.editage.jp) for English language editing. We would like to thank JCOG Bone and Soft Tissue Tumor Study Group. Participating Institutions of JCOG0304 were as follows (from north to south): Sapporo Medical University, Hokkaido Cancer Center, Tohoku University, Chiba Cancer Center, National Cancer Center Hospital, Nihon University, Kyorin University, Keio University, Cancer Institute Hospital, Teikyo University, Kanagawa Cancer Center, Niigata Cancer Center Hospital, Kanazawa University, University of Yamanashi, Gifu University, Shizuoka Cancer Center, Mie University, Kyoto University, Osaka University, Osaka International Cancer Center, Tottori University, Okayama University, Hiroshima Prefectural Hospital, Kanmon Medical Center, Kyushu University, National Kyushu Cancer Center.
\end{abstract}

\section{Authors' contributions}

$\mathrm{KT}, \mathrm{JM}, \mathrm{HF}$ and $\mathrm{Yl}$ conceived the study concept and design. JM and HF performed statistical analysis and interpretation of data. KT, NN, AK, HK, TH, YM, HT, RN, HH, ME, MW, YY, TO, SA, KA, RY, HH, TY, TM, KA, AN, HY and TO carried out patient accrual and acquisition, analysis and interpretation of data. $\mathrm{KT}, \mathrm{JM}, \mathrm{HHi}, \mathrm{HF}, \mathrm{TO}$ and $\mathrm{YI}$ conducted drafting and revision of the manuscript. All authors have read and approved the final manuscript. 


\section{Funding}

This work was supported in part by the National Cancer Center Research and Development Fund [grant number 29-A-3] and AMED [grant number JP18ck0106336] for the collection, analysis, and interpretation of data and for preparing the manuscript.

\section{Availability of data and materials}

Individual participant data that underlie the results reported in this Article, after deidentification, will be shared with investigators whose proposed use of the data has been approved by the investigators from the Bone and Soft Tissue Tumor Study Group of the Japan Clinical Oncology Group. Proposals should be directed to the corresponding author.

\section{Ethics approval and consent to participate}

The study protocol was approved by the Protocol Review Committee of JCOG, and was also approved by the Institutional Review Boards of each of the 27 participating institutions (Additional file 1: Table S1). All patients provided written informed consent before entry into the study.

\section{Consent for publication}

All patients provided written informed consent for publication before entry into the study.

\section{Competing interests}

The authors declare that they have no competing interests.

\section{Author details}

'Department of Orthopaedic Surgery, Faculty of Medicine, Oita University, Idaigaoka 1-1, Hasama, Yufu City, Oita 879-5593, Japan. JCOG Data Center, National Cancer Center Hospital, Tokyo 104-0045, Japan. ${ }^{3}$ Musculoskeletal Oncology Service, Osaka International Cancer Institute, Osaka 541-8567, Japan. ${ }^{4}$ Department of Orthopaedic Surgery, National Cancer Center, Tokyo 104-0045, Japan. ${ }^{5}$ Department of Orthopaedic Surgery, Shizuoka Cancer Center, Shizuoka 411-0934, Japan. 'Department of Orthopaedic Surgery, Kanagawa Cancer Center, Kanagawa 241-0815, Japan. ${ }^{7}$ Department of Orthopaedic Surgery, Kyushu University, Fukuoka 812-8582, Japan. ${ }^{8}$ Department of Orthopaedic Surgery, Kanazawa University, Ishikawa 920-8641, Japan. ${ }^{9}$ Department of Orthopaedic Surgery, Keio University, Tokyo 160-0016, Japan. ${ }^{10}$ Department of Orthopaedic Surgery, Niigata Cancer Center Hospital, Niigata 951-8133, Japan. ${ }^{11}$ Department of Orthopaedic Surgery, Sapporo Medical University, Sapporo 060-8556, Japan. ${ }^{12}$ Department of Orthopaedic Surgery, Tohoku University, Sendai 980-8575, Japan. ${ }^{13}$ Department of Orthopaedic Surgery, Nihon University, Tokyo 173-8610, Japan. ${ }^{14}$ Department of Orthopaedic Surgery, Kyoto University, Kyoto 606-8501, Japan. ${ }^{15}$ Department of Orthopaedic Surgery, Teikyo University, Tokyo 173-8606, Japan. ${ }^{16}$ Department of Orthopaedic Surgery, Mie University, Mie 514-8507, Japan. ${ }^{17}$ Department of Orthopaedic Surgery, National Kyushu Cancer Center, Fukuoka 811-1395, Japan. ${ }^{18}$ Department of Orthopaedic Surgery, Hokkaido Cancer Center, Sapporo 003-0804, Japan. ${ }^{19}$ Department of Orthopaedic Surgery, Chiba Cancer Center, Chiba 260-8717, Japan. ${ }^{20}$ Department of Orthopaedic Surgery, Kyorin University, Tokyo 181-8611, Japan. ${ }^{21}$ Department of Orthopaedic Surgery, Cancer Institute Hospital, Tokyo 135-8550, Japan. ${ }^{22}$ Department of Orthopaedic Surgery, Gifu University, Gifu 501-1194, Japan. ${ }^{23}$ Department of Orthopaedic Surgery, Osaka University, Osaka 565-0871, Japan. ${ }^{24}$ Department of Orthopaedic Surgery, Okayama University, Okayama 700-0914, Japan. ${ }^{25}$ Kyushu Rosai Hospital, Kitakyushu 800-0296, Japan.

Received: 27 May 2019 Accepted: 30 August 2019

Published online: 06 September 2019

\section{References}

1. American Cancer Society. Cancer facts and figures 2019. American Cancer Society. 2019. https://www.cancer.org/cancer/all-cancer-types.html. .

2. Japanese Orthopaedic Association Musculoskeletal Tumor Committee. Soft tissue tumor registry in Japan 2015. Tokyo: National Cancer Center; 2015

3. Casali PG, Abecassis N, Aro HT, Bauer S, Biagini R, Bielack S, et al. Soft tissue and visceral sarcomas: ESMO-EURACAN clinical practice guidelines for diagnosis, treatment and follow-up. Ann Oncol. 2018;29:iv268-9.

4. Tanaka K, Kawamoto H, Saito I, Yoshimura K, Fukuda H, Iwamoto Y. Preoperative and postoperative chemotherapy with ifosfamide and adriamycin for adult high-grade soft-tissue sarcomas in the extremities: Japan clinical oncology group study JCOG0304. Jpn J Clin Oncol. 2009;39:271-3.

5. Tanaka K, Mizusawa J, Fukuda H, Araki N, Chuman H, Takahashi M, et al. Perioperative chemotherapy with ifosfamide and doxorubicin for highgrade soft tissue sarcomas in the extremities (JCOG0304). Jpn J Clin Oncol. 2015;45:555-61.

6. Tanaka K, Hasegawa T, Nojima T, Oda Y, Mizusawa J, Fukuda H, et al. Prospective evaluation of Ki-67 system in histological grading of soft tissue sarcomas in the Japan clinical oncology group study JCOG0304. World J Surg Oncol. 2016;14:110.

7. Tanaka K, Ogawa G, Mizusawa J, Naka N, Kawai A, Takahashi M, et al. Prospective comparison of various radiological response criteria and pathological response to preoperative chemotherapy and survival in operable high-grade soft tissue sarcomas in the Japan clinical oncology group study JCOG0304. World J Surg Oncol. 2018;16:162.

8. International Union Against Cancer. TNM classification of malignant tumors, sixth edition. Sobin LH, Wittekind CH eds. New York: Wiley-Liss Inc., Kanehara Co Ltd; 2003. p. 117-21.

9. Guillou L, Coindre JM, Bonichon F, Nguyen BB, Terrier P, Collin F, et al. Comparative study of the National Cancer Institute and French Federation of Cancer Centers Sarcoma Group grading systems in a population of 410 adult patients with soft tissue sarcoma. J Clin Oncol. 1997;15:350-62.

10. Gronchi A, Stacchiotti S, Verderio P, Ferrari S, Martin Broto J, Lopez-Pousa A, et al. Short, full-dose adjuvant chemotherapy in high-risk adult soft tissue sarcomas: long-term follow-up of a randomized clinical trial from the Italian sarcoma group and the Spanish sarcoma group. Ann Oncol. 2016;27:2283-8.

11. Issels RD, Lindner LH, Verweij J, Wessalowski R, Reichardt P, Wust P, et al. Effect of neoadjuvant chemotherapy plus regional hyperthermia on long-term outcomes among patients with localized high-risk soft tissue sarcoma: the EORTC 62961-ESHO 95 randomized clinical trial. JAMA Oncol. 2018;4:483-92.

12. Frustaci S, Gherlinzoni F, De Paoli A, Bonetti M, Azzarelli A, Comandone A, et al. Adjuvant chemotherapy for adult soft tissue sarcomas of the extremities and girdles: results of the Italian randomized cooperative trial. J Clin Oncol. 2001;19:1238-47.

13. Frustaci S, De Paoli A, Bidoli E, La Mura N, Berretta M, Buonadonna A, et al. Ifosfamide in the adjuvant therapy of soft tissue sarcomas. Oncology. 2003;65:80-4.

14. Gronchi A, Frustaci S, Mercuri M, Martin J, Lopez-Pousa A, Verderio P, et al. Short, full-dose adjuvant chemotherapy in high-risk adult soft tissue sarcomas: a randomized clinical trial from the Italian sarcoma group and the Spanish sarcoma group. J Clin Oncol. 2012;30:850-6.

15. Gronchi A, Ferrari S, Quagliuolo V, Broto JM, Pousa AL, Grignani G, et al. Histotype-tailored neoadjuvant chemotherapy versus standard chemotherapy in patients with high-risk soft-tissue sarcomas (ISG-STS 1001): an international, open-label, randomised, controlled, phase 3, multicentre trial. Lancet Oncol. 2017;18:812-22.

16. Penaiz N, Colterjohn N, Farrokhyar F, Tozer R, Figueredo A, Ghert M. A systematic meta-analysis of randomized controlled trials of adjuvant chemotherapy for localized resectable soft-tissue sarcoma. Cancer. 2008;113:573-81.

17. Woll PJ, Reichardt P, Le Cesne A, Bonvalot S, Azzarelli A, Hoekstra HJ, et al. Adjuvant chemotherapy with doxorubicin, ifosfamide, and lenograstim for resected soft-tissue sarcoma (EORTC 62931): a multicentre randomised controlled trial. Lancet Oncol. 2012;13:1045-54.

18. Kataoka K, Tanaka K, Mizusawa J, Kimura A, Hiraga H, Kawai A, et al. A randomized phase $\|/\| I$ trial of perioperative chemotherapy with adriamycin plus ifosfamide versus gemcitabine plus docetaxel for high-grade soft tissue sarcoma: Japan clinical oncology group study JCOG1306. Jpn J Clin Oncol. 2014;44:765-9.

19. Tanaka K, Mizusawa J, Naka N, Kawai A, Katagiri H, Hiruma T, et al. 10-year follow-up results of perioperative chemotherapy with doxorubicin (DXR) and ifosfamide (IFM) for high-grade soft tissue sarcomas (STS) in the extremities: Japan clinical oncology group study JCOG0304. J Clin Oncol. 2019;37(suppl): e22533 http://abstracts.asco.org/239/AbstView_239_257101.html.

\section{Publisher's Note}

Springer Nature remains neutral with regard to jurisdictional claims in published maps and institutional affiliations. 